\title{
PAPER
}

\section{Multicritical points in a model for $5 f$-electron systems under pressure and magnetic field}

To cite this article: Julián Faúndez et al 2021 J. Phys.: Condens. Matter 33295801

View the article online for updates and enhancements.

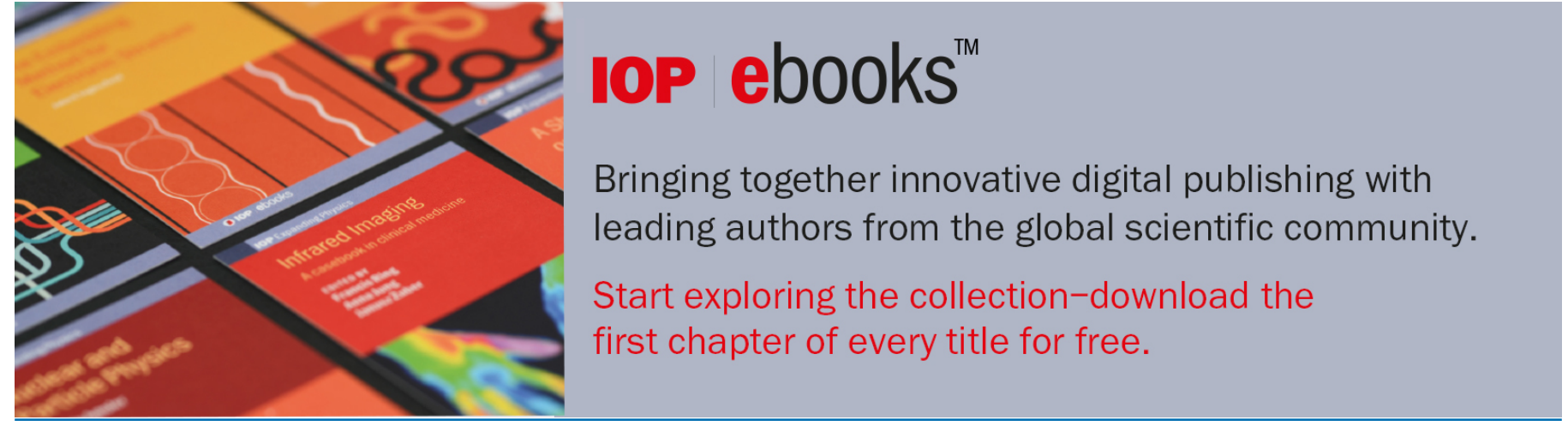

This content was downloaded from IP address 200.130 .19 .173 on $01 / 09 / 2021$ at $14: 41$ 


\title{
Multicritical points in a model for $5 f$-electron systems under pressure and magnetic field
}

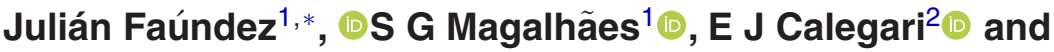 \\ P S Riseborough ${ }^{3}$ (1)
}

\footnotetext{
${ }^{1}$ Instituto de Física, Universidade Federal do Rio Grande do Sul, 91501-970 Porto Alegre, RS, Brazil 2 Departamento de Física - Universidade Federal de Santa Maria, 97105-900, Santa Maria, RS, Brazil

3 Physics Department, Temple University, Philadelphia, PA 19122, United States of America
}

E-mail: julian.faundez@ufrgs.br

Received 5 November 2020, revised 19 January 2021

Accepted for publication 9 February 2021

Published 4 June 2021

\begin{abstract}
We investigate the evolution of multicritical points under pressure and magnetic field in a model described by two $5 f$ bands (called $\alpha$ and $\beta$ ) that hybridize with a single itinerant conduction band. The interaction is given by the direct Coulomb and the Hund's rule exchange terms. Three types of orderings are considered: two conventional spin density waves (SDWs) and an exotic SDW, i.e., with no magnetic moment formation. The conventional SDWs phases, are characterized by $m_{f}^{\beta}>m_{f}^{\alpha}$ and $m_{f}^{\alpha}>m_{f}^{\beta}$, respectively, where $m_{f}^{\alpha}$ and $m_{f}^{\beta}$ are the intraband staggered magnetizations. The exotic SDW, which has time reversal symmetry, is described by a purely imaginary order parameter. This phase is related to a band mixing given by the spin-flip part of the Hund's rule exchange interaction. As result, without magnetic field, the phase diagrams of temperature $(T)$ versus pressure (given by the variation of the bandwidth $(W)$ ) shows a sequence of phase transitions involving the three phases which gives rise to multicritical points. The presence of the magnetic field $\left(h_{z}\right)$ has drastic effects on part of the phase diagram and the location of the multicritical points.
\end{abstract}

Keywords: multicritical points, convencional and exotic SDW, 5- $f$ electrons

(Some figures may appear in colour only in the online journal)

\section{Introduction}

The $5 f$ electron systems with their multiplicity of states of matter, conventional and unconventional, competing for stability is a natural ground for the presence of classical and quantum multicritical points. Recently, there have been several observations in these systems that indicate classical tricritical points (TCPs) as, for instance, $\mathrm{USb}_{2}$ [1], $\mathrm{UN}$ [2], $\mathrm{UAu}_{2} \mathrm{Si}_{2}$ [3] and $\mathrm{URu}_{2} \mathrm{Si}_{2}$ [4] when a magnetic field is applied. An other example is the presence of a classical bicritical point (BCP) that appears in $\mathrm{URu}_{2} \mathrm{Si}_{2}$ when hydrostatic pressure is applied, which is related to the competition between the puzzling hidden order and an antiferromagnetic phase [5]. The presence of

* Author to whom any correspondence should be addressed. classical multicritical points allows a very interesting development. They may eventually evolve, by varying some intensive parameter, to become quantum ones [6] and thus, possibly, exhibit behavior that deviates from the standard Fermi liquid $[7,8]$. Even so, the topic of classical multicritical points in the physics of $5 f$ electron systems has not yet received due attention.

The first question that may be asked is whether and which Anderson-type model would be suitable to describe the phase competition found in $5 f$ electron systems. Very recently, the so called underscreened Anderson lattice model (UALM) has been used to study multicritical points arising from competition between conventional itinerant antiferromagnetic phases [9]. This model has two degenerate narrow $5 f$ bands (labeled by $\chi=\alpha, \beta)$, which are hybridized with a single itinerant 
conduction band. The interaction is composed of the Coulomb and the Hund's rule exchange interactions. This last term makes the model manifestly spin-rotational invariant [10]. Indeed, the UALM has a close relationship with the underscreened Kondo lattice model via a Schrieffer-Wolff transformation. This last model successfully described the coexistence of ferromagnetism and Kondo effect observed in uranium and neptunium compounds (see references [11, 12] and references there in). Since the UALM can be considered a generalization of the undrescreened Kondo lattice model, it might also be considered appropriate to describe some aspects of the $5 f$ electron systems.

Our goal in this work is to show how classical multicritical points can emerge from the competition between conventional and unconventional spin density waves (SDWs) hosted in the UALM. In fact, we refer to SDWs given by order parameters (OPs) with odd or even parity under time reversal symmetry. For the last case, we highlight the role of the Hund's rule exchange interaction in the UALM which gives a particular type of mixing of the two $5 f$ bands, allowing the model to host a phase that break spin-rotational and space-translational symmetry but does not the time reversal one. More precisely, this exotic SDW, which does not involve magnetic moment formation, is specifically related to the spin-flip part of Hund's rule exchange interaction. We remark that this non-magnetic SDW has been proposed to describe the hidden order in $\mathrm{URu}_{2} \mathrm{Si}_{2}$ [13]. From now on, we refer to this phase as an inter-orbital spin density wave (IOSDW). In addition, there are two distinct intraband SDWs which can be stabilized with spin gap at the same ordering wave vector $[9,13]$.

Specificaly, we study here, within the mean field approximation, the competition between the IOSDW and the conventional SDWs in phase diagrams where pressure and magnetic field are applied simultaneously. For this purpose, we explore a scenario where the instability of the paramagnetic phase toward to conventional and non-conventional SDWs occurs at the same nesting vector. Here, we assume that the applied pressure changes the inter-atomic distances and, thereby, changes the bandwidth $(W)$. In fact, $W$ is the only model parameter to be changed in the present work. The hybridization remains constant. Furthermore, the hybridization matrix elements are $\vec{k}$-independent. As a consequence, one may transform the basis of the $5 f$ states into a new basis in which a linear combination of $f$ orbitals hybridize and the remaining orthogonal states do not. This can lead to a situation which breaks the symmetry between the $5 f$ bands. In other words, when the bandwidth $W$ increases, the intra-band nesting may occur simultaneously for both bands or one band may become depart from the perfect nesting condition. Finally, we consider that the conventional SDWs has a Neel OP which is fixed by an Ising-like anisotropy as found in some uranium compounds [14-16]. The magnetic field is applied longitudinally to the Ising-like anisotropy.

Some consequences competition between the phases described above can be ascertained in advance from general arguments based on a Landau free-energy expansion with more than one OP. The simplest case with two OPs which have odd parity, their coupling should be linear in a Landau free-energy expansion. Since there is no additional change in symmetry between the two phases, there is a first-order transitions between them ending at a critical end point [17]. Within UALM, these SDWs are characterized by a staggered magnetization for each band (here called $\alpha$ or $\beta$ ) given by $m_{f}^{\alpha}$ and $m_{f}^{\beta}$. The first SDW, called $\mathrm{AF}_{1}$, occurs when $m_{f}^{\beta}>m_{f}^{\alpha}$, while in the other one, called $\mathrm{AF}_{2}$, when $m_{f}^{\alpha}>m_{f}^{\beta}$. Indeed, the prediction of the existence of a critical end point has been confirmed in the UALM [9]. In the case where IOSDW is also stabilized, the coupling among OPs is more complicated since they have distinct parity properties under time reversal symmetry [18]. Therefore, in terms of a Landau free energy expansion, the competition among $\mathrm{AF}_{1}, \mathrm{AF}_{2}$ and $\mathrm{IOSDW}$ can lead to a $\mathrm{BCP}$ or a tetracritical point [19]. For further variation of pressure or field, the nesting condition may no longer be satisfied leading to the suppression of the ordered phases. This last phase transition line can present a TCP [9].

Lastly, because of the asymmetry between the bands, we remark that the simultaneous effects of pressure the magnetic field on the phases $\mathrm{AF}_{1}, \mathrm{AF}_{2}$, IOSDW and, consequently on the multicritical points, are closely connected with changes in the electronic structure of the problem. Particularly, the effects of the magnetic field on each of the phases can be traced directly from changes in the quasiparticle dispersion relations [20].

This paper is organized as follow: the UALM is presented in the section 2. In the section subsequent, we derive the Green's functions and the free energy. Then, in section 4, we introduce the OPs to describe the usual and the exotic phases that appear in the problem. In section 5, we present the phase diagrams temperature vs pressure without and with magnetic field as well as results the electronic structure. Section 6 is dedicated to the multicritical points and their evolution under magnetic field. The conclusions and other remarks are found in section 7 .

\section{Model}

The UALM Hamiltonian consists of three terms

$$
\hat{H}=\hat{H}_{f}+\hat{H}_{d}+\hat{H}_{f d} .
$$

The $5 f$-electron term $\hat{H}_{f}$ is split in two parts as

$$
\hat{H}_{f}=\hat{H}_{f, 0}+\hat{H}_{f, 1}
$$

where the free part $H_{0, f}$ describes two degenerate narrow $5 f$ bands as given below

$$
\hat{H}_{f, 0}=\sum_{\vec{k}, \sigma} \sum_{\chi} E_{f}^{\chi}(\vec{k}) f_{\vec{k}, \sigma}^{\dagger \chi} f_{\vec{k}, \sigma}^{\chi} .
$$

The $f_{\vec{k}, \sigma}^{\dagger \chi}$ are the creation operators for electrons with spin $\sigma(=\uparrow, \downarrow)$ at site $i$. The $\chi$-bands $(\chi=\alpha$ and $\beta$ ) in equation (3) follow the intraband and interband nesting property $E_{f}^{\chi}(\vec{k}+$ $\vec{Q})=-E_{f}^{\chi^{\prime}}(\vec{k})$ where $\chi=\chi^{\prime}$ or $\chi \neq \chi^{\prime} . E_{f}^{\chi}(\vec{k})$ describes an electron band dispersion relation and the vector $\vec{Q}$ is a commensurate momentum transfer in the first Brillouin zone. 
The second term in equation (2) describes the local Coulomb and Hund's rule exchange interactions given as

$$
\begin{aligned}
\hat{H}_{f, 1}= & \left(\frac{U}{2 N}\right) \sum_{\vec{k}, \vec{k}^{\prime}, \vec{q}, \sigma, \sigma^{\prime}, \chi, \chi^{\prime}} f_{\vec{k}+\vec{q}, \sigma}^{\dagger, \chi} f_{\vec{k}^{\prime}-\vec{q}, \sigma^{\prime}}^{\dagger, \chi^{\prime}} f_{\vec{k}, \sigma^{\prime}}^{\chi^{\prime}} f_{\vec{k}^{\prime}, \sigma}^{\chi} \\
& +\left(\frac{J}{2 N}\right) \sum_{\vec{k}, \vec{k}^{\prime}, \vec{q}, \sigma, \sigma^{\prime}, \chi, \chi^{\prime}} f_{\vec{k}+\vec{q}, \sigma}^{\dagger, \chi} f_{\vec{k}^{\prime}-\vec{q}, \sigma^{\prime}}^{\dagger, \chi^{\prime}} f_{\vec{k}^{\prime}, \sigma^{\prime}}^{\chi} f_{\vec{k}, \sigma}^{\chi^{\prime}} .
\end{aligned}
$$

The conduction electron term $\hat{H}_{d}$ is expressed as

$$
\hat{H}_{d}=\sum_{\vec{k}, \sigma} \epsilon_{d}(\vec{k}) d_{\vec{k}, \sigma}^{\dagger} d_{\vec{k}, \sigma}
$$

where $\epsilon(\vec{k})$ describes the dispersion relation of conduction electrons labeled by the Bloch wave vector $\vec{k}$. The last term in equation (1) describes the on-site hybridization process in the UAL model by

$$
\hat{H}_{f d}=\sum_{\vec{k}, \sigma} \sum_{\chi=\alpha \beta}\left(V_{\chi}(\vec{k}) f_{\vec{k}, \sigma}^{\dagger \chi} d_{\vec{k}, \sigma}+V_{\chi}^{*}(\vec{k}) d_{\vec{k}, \sigma}^{\dagger} f_{\vec{k}, \sigma}^{\chi}\right) .
$$

The applied magnetic field can be added to equation (1) is given as

$$
\hat{H}_{\mathrm{ext}}=-\sum_{\vec{k}} \sum_{\sigma= \pm} \sigma\left[H_{z}^{f} f_{\vec{k}, \sigma}^{\dagger} f_{\vec{k}, \sigma}+H_{z}^{d} d_{\vec{k}, \sigma}^{\dagger} d_{\vec{k}, \sigma}\right]
$$

with $H_{z}^{f(d)}=g_{f(d)} \mu_{\mathrm{B}} h_{z}$. The value $\sigma=1$ and -1 correspond to the up and down spin projections, respectively.

In the present work, the $5 f$ band energy $E_{f}^{\chi}(\vec{k})=\epsilon_{f}+\epsilon_{f}(\vec{k})$ and the conduction one $\epsilon_{d}(\vec{k})$ pertain to a cubic lattice, thus

$$
\epsilon_{b}(\vec{k})=-2 t_{b}\left[\cos \left(k_{x} a\right)+\cos \left(k_{y} a\right)+\cos \left(k_{z} a\right)\right]
$$

in which $b=f$ or $d$, and $a$ is the lattice parameter.

We apply a mean field approximation to the fluctuations of the $f$-electrons operators that produces two possible instabilities of the normal-paramagnetic phase in the UAL model, i.e., the IOSDW and the itinerant antiferromagnetic phase. Therefore, we consider the normalized operators below related to each instability:

$$
\hat{z}_{\vec{q}, \sigma}^{\chi^{\prime} \chi}=\frac{1}{N} \sum_{\vec{k}} f_{\vec{k}+\vec{q}, \sigma}^{\dagger, \chi^{\prime}} f_{\vec{k}, \sigma}^{\chi}\left(\chi \neq \chi^{\prime}\right)
$$

and

$$
\hat{n}_{\vec{q}, \sigma}^{\chi \chi}=\frac{1}{N} \sum_{\vec{k}} f_{\vec{k}+\vec{q}, \sigma}^{\dagger, \chi} f_{\vec{k}, \sigma}^{\chi} .
$$

Thus, the interaction term of the Hamiltonian given in the equation (4) is expanded in powers of

$$
\Delta \hat{z}_{\vec{q}, \sigma}^{\chi \chi^{\prime}}=\hat{z}_{\vec{q}, \sigma}^{\chi \chi^{\prime}}-z_{\vec{q}, \sigma}^{\chi \chi^{\prime}}
$$

and

$$
\Delta \hat{n}_{\vec{q}, \sigma}^{\chi \chi}=\hat{n}_{\vec{q}, \sigma}^{\chi \chi}-n_{\vec{q}, \sigma}^{\chi \chi} .
$$

Therefore, the IOSDW OP is given by the expectation value $z_{\vec{q}, \sigma}^{\chi^{\prime} \chi}$. The staggered magnetizations for each $f$-band, $m_{f}^{\alpha}$ and $m_{f}^{\beta}$ are obtained from

$$
m_{f}^{\chi}=\frac{1}{2}\left(n_{\vec{Q}, \uparrow}^{\chi \chi}-n_{\vec{Q}, \downarrow}^{\chi \chi}\right)
$$

\section{Green's functions}

We assume the intra-orbital SDWs and the IOSDW instabilities occur at the same nesting vector $\vec{Q}$ of the cubic lattice. Therefore, for the expectations values in equation (11) and (12), we make the ansatz:

$$
z_{\vec{q}, \sigma}^{\chi^{\prime} \chi}=z_{\vec{Q}, \sigma}^{\chi^{\prime} \chi} \delta_{\vec{q}, \vec{Q}}
$$

and

$$
n_{\vec{q}, \sigma}^{\chi \chi}=n_{\sigma}^{\chi \chi} \delta_{\vec{q}, 0}+n_{\vec{Q} \sigma}^{\chi \chi} \delta_{\vec{q}, \vec{Q}} .
$$

From now on, we follow closely references [9, 13] choosing a basis set for the $f$ orbitals as $V_{\beta}(\vec{k})=0$ and $V_{\alpha}(\vec{k})=V_{\alpha}$. The temporal and spatial Fourier transform of the single-electron $f-f$ Green's functions is completed with the mixed $f-d$ Green's function equation of motion given a closed set which can be solved within a matrix formalism. Thus, one has

$$
\underline{G}_{\vec{Q}, \sigma}\left(\vec{k}, \overrightarrow{k^{\prime}}, \omega\right)=\left(\Upsilon_{\vec{Q}, \sigma}(\vec{k}, \omega)\right)^{-1} \underline{\delta}_{\vec{Q}}\left(\vec{k}, \overrightarrow{k^{\prime}}\right)
$$

with

$$
\underline{G}_{\vec{Q}, \sigma}\left(\vec{k}, \overrightarrow{k^{\prime}}, \omega\right)=\left(\begin{array}{c}
G_{f f, \sigma}^{\alpha \chi^{\prime}}\left(\vec{k}, \overrightarrow{k^{\prime}}, \omega\right) \\
G_{f f, \sigma}^{\beta \chi^{\prime}}\left(\vec{k}, \overrightarrow{k^{\prime}}, \omega\right) \\
G_{f f, \sigma}^{\alpha \chi^{\prime}}\left(\vec{k}+\vec{Q}, \overrightarrow{k^{\prime}}, \omega\right) \\
G_{f f, \sigma}^{\beta \chi^{\prime}}\left(\vec{k}+\vec{Q}, \overrightarrow{k^{\prime}}, \omega\right)
\end{array}\right)
$$

and

$$
\underline{\delta}_{\vec{Q}}\left(\vec{k}, \overrightarrow{k^{\prime}}\right)=\left(\begin{array}{c}
\delta^{\alpha \chi^{\prime}} \delta_{\vec{k}, \vec{k}^{\prime}} \\
\delta^{\beta \chi^{\prime}} \delta_{\vec{k}, \overrightarrow{k^{\prime}}} \\
\delta^{\alpha \chi^{\prime} \delta_{\vec{k}+\vec{Q}, \overrightarrow{k^{\prime}}}} \\
\delta^{\beta \chi^{\prime} \delta_{\vec{k}+\vec{Q}, \overrightarrow{k^{\prime}}}}
\end{array}\right)
$$

The matrix $\Upsilon_{\vec{Q}, \sigma}(\vec{k}, \omega)$ is defined below

$$
\underline{\Upsilon}_{\vec{Q}, \sigma}(\vec{k}, \omega)=\omega \underline{I}-\left(\begin{array}{cc}
\underline{\Lambda}_{\sigma}(\vec{k}, \omega) & -\underline{\Phi}-\vec{Q}, \sigma \\
-\underline{\Phi}_{-\vec{Q}, \sigma}^{\dagger} & \underline{\Lambda}_{\sigma}(\vec{k}+\vec{Q}, \omega)
\end{array}\right)
$$

where $\underline{I}$ is the unit matrix,

$$
\underline{\Lambda}_{\sigma}(\vec{k}, \omega)=\left(\begin{array}{cc}
E_{f \sigma}^{\alpha}(\vec{k})-\xi^{\alpha}(\vec{k}, \omega) & 0 \\
0 & E_{f \sigma}^{\beta}(\vec{k})-\xi^{\beta}(\vec{k}, \omega)
\end{array}\right)
$$

and

$$
\underline{\Phi}_{-\vec{Q}, \sigma}=\left(\begin{array}{cc}
-\phi_{-\vec{Q}, \sigma}^{\alpha \alpha} & -\kappa_{-\vec{Q}, \sigma}^{\beta \alpha} \\
-\kappa_{-\vec{Q}, \sigma}^{\alpha \beta} & -\phi_{-\vec{Q}, \sigma}^{\beta \beta}
\end{array}\right) .
$$

In the matrix $\underline{\Lambda}_{\sigma}(\vec{k}, \omega)$, the mean field dispersion relation $\tilde{E}_{f}^{\chi}(\vec{k})$ is given by 


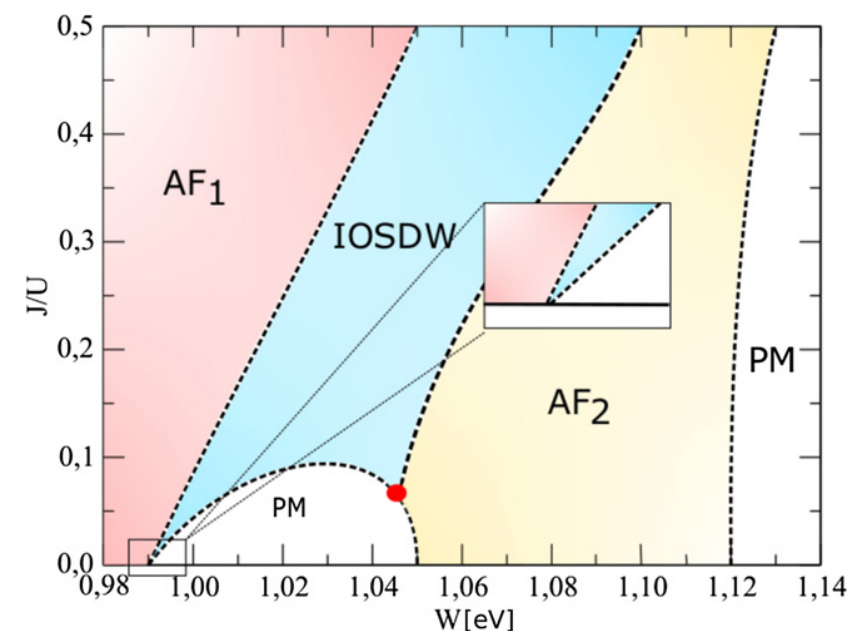

Figure 1. The phase diagram for $J / U$ versus $W$ for $T=0$. The red point represents a triple point. There is no triple point in the inset (see discussion in the text)

$$
\begin{aligned}
E_{f \sigma}^{\chi}(\vec{k})= & E_{f}^{\chi}(\vec{k})-\sigma H_{z}^{f} \\
& +\sum_{\chi^{\prime}}\left(U n_{-\sigma}^{\chi^{\prime} \chi^{\prime}}+(U-J) n_{\sigma}^{\chi^{\prime} \chi^{\prime}}\left(1-\delta^{\chi, \chi^{\prime}}\right)\right) .
\end{aligned}
$$

One also has

$$
\xi^{\chi}(\vec{k}, \omega)=\frac{\left|V_{\chi}\right|^{2}}{\omega-\epsilon_{d \sigma}(\vec{k})}\left[\delta_{\chi \alpha}+\left(1-\delta_{\chi \beta}\right)\right]
$$

with $\epsilon_{d \sigma}(\vec{k})=\epsilon(\vec{k})-\sigma H_{z}^{d}$. The gaps $\kappa_{-\underline{Q}, \sigma}^{\chi^{\prime} \chi}$ and $\phi_{-\vec{Q}, \sigma}^{\chi \chi}$ in the matrix $\underline{\Sigma}_{\vec{Q}, \sigma}$ are given as

$$
\kappa_{-\vec{Q}, \sigma}^{\chi^{\prime} \chi}=J z_{\vec{Q},-\sigma}^{\chi^{\prime} \chi}-(U-J) z_{\vec{Q}, \sigma}^{\chi^{\prime} \chi}
$$

and

$$
\phi_{-\vec{Q} \sigma}^{\chi \chi}=\sum_{\chi^{\prime}}\left(U n_{\vec{Q},-\sigma}^{\chi^{\prime} \chi^{\prime}}+(U-J) n_{\vec{Q}, \sigma}^{\chi^{\prime} \chi^{\prime}}\left(1-\delta^{\chi, \chi^{\prime}}\right)\right) .
$$

\section{OPs and free energy}

In this section, we discuss the OPs for the competing conventional and unconventional SDWs.

The IOSDW OP is given by the expectation value of the non-Hermitian operator given in equation (9). Thus :

$$
z_{-\vec{Q}, \sigma}^{\alpha \beta}=\frac{1}{N} \sum_{\vec{k}, \sigma} \int_{C} \frac{\mathrm{d} \omega}{2 \pi i} f(\omega) G_{f, \sigma}^{\beta \alpha}(\vec{k}, \vec{k}+\vec{Q}, \omega),
$$
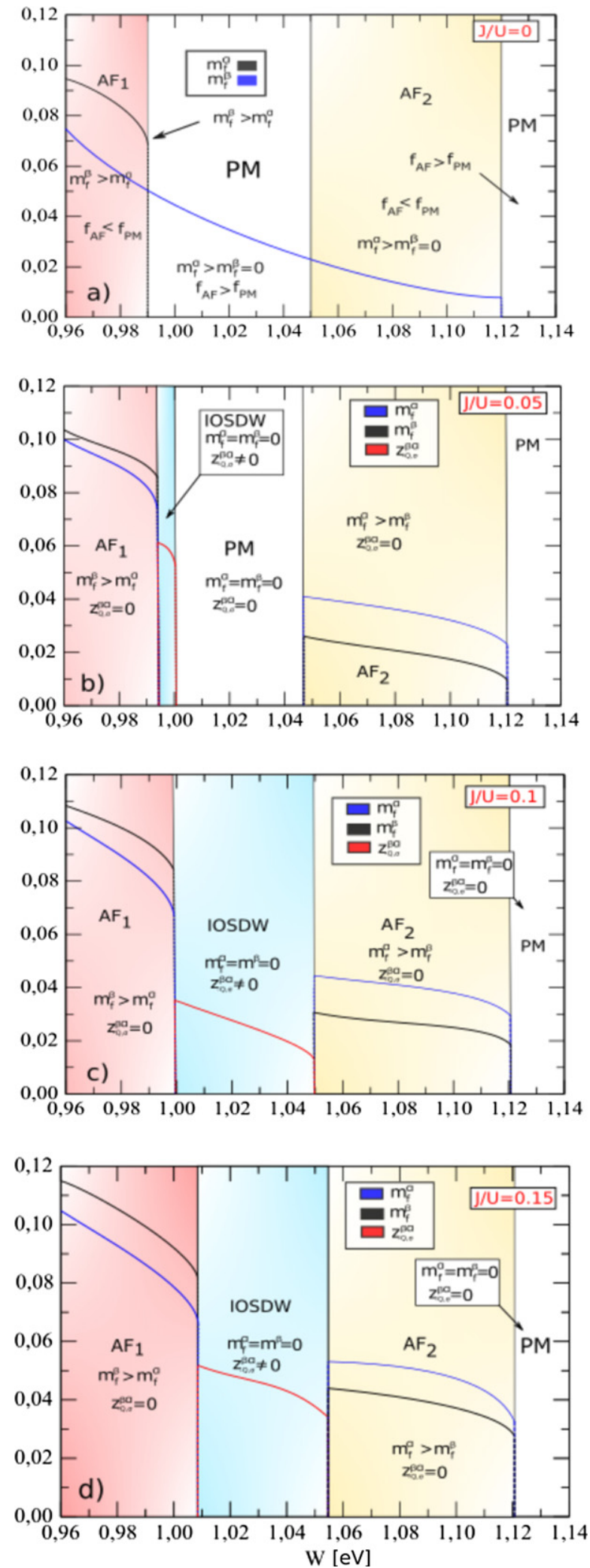

Figure 2. Behavior of the OPs as function of $W$ for different values of $J / U$ at $T=0$. 


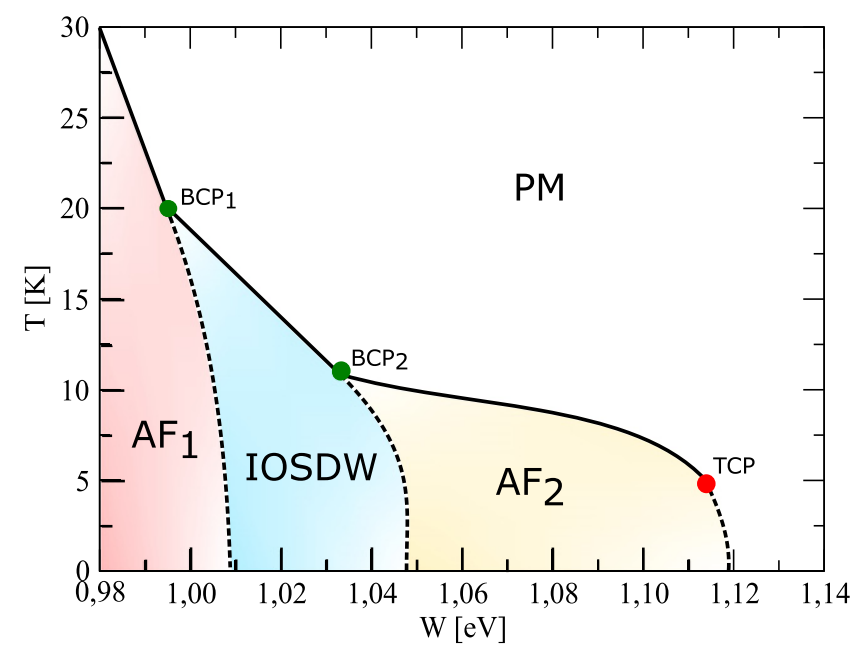

Figure 3. Phase diagram of $T$ versus $W$. The continuous line represents a second order transition while the dotted line is a first-order transition. The two green points are BCPs and the red point is a TCP.

where $f(\omega)$ is the Fermi function and $G_{f, \sigma}^{\beta \alpha}(\vec{k}, \vec{k}+\vec{Q}, \omega)$ is given in equation (A.1). The integration contour closes the real axis and does not include the poles of the Fermi-Dirac distribution. We can re-write equation (24) as

$$
z_{-\vec{Q}, \sigma}^{\alpha \beta}=\kappa_{-\vec{Q}, \sigma}^{\beta \alpha} X_{1 \sigma}(\vec{Q})+\phi_{-\vec{Q} \sigma}^{\alpha \alpha} \phi_{-\vec{Q} \sigma}^{\beta \beta} X_{2 \sigma}(\vec{Q})
$$

where

$$
\begin{aligned}
X_{1 \sigma}(\vec{Q})= & \frac{1}{N} \sum_{\vec{k}} \int_{C} \frac{\mathrm{d} \omega}{2 \pi i} f(\omega) \\
& \times \frac{g_{\sigma}^{\alpha}(\vec{k}, \omega) g_{\sigma}^{\beta}(\vec{k}+\vec{Q}, \omega)-\left|\kappa_{-\vec{Q}, \sigma}^{\beta \alpha}\right|^{2}}{D_{\vec{Q} \sigma}(\vec{k}, \omega)}
\end{aligned}
$$

and

$$
X_{2 \sigma}(\vec{Q}, \sigma)=\frac{1}{N} \sum_{\vec{k}} \int_{C} \frac{\mathrm{d} \omega}{2 \pi i} \frac{f(\omega)}{D_{\vec{Q} \sigma}(\vec{k}, \omega)}
$$

with $D_{\vec{Q}_{\sigma}}(\vec{k}, \omega)$ defined in equation (A.4). Moreover,

$$
g_{\sigma}^{\chi}(\omega, \vec{k})=\left(\omega-E_{f \sigma}^{\chi}(\vec{k})-\xi^{\chi}(\vec{k}, \omega)\right)
$$

where $\xi(\vec{k}, \omega)$ is given in equation (21). From equations (22) and (25), one can see that $z_{-\vec{Q}, \sigma}^{\beta \alpha}$ and $z_{-\vec{Q},-\sigma}^{\beta \alpha}$ are coupled by the Hund's rule exchange interaction. Actually, the IOSDW solution implies that $z_{-\vec{Q}, \sigma}^{\beta \alpha}=-z_{-\vec{Q},-\sigma}^{\beta \alpha}$. Therefore, for IOSDW to be time reversal invariant, which is the reason for its nonmagnetic character, the OP needs to be a purely imaginary quantity $[13,20]$.

The real staggered magnetizations with Ising anisotropy $m_{f}^{\chi}(\chi=\alpha$ and $\beta)$ (see equation (13)) are obtained from the Green's function $G_{f, \sigma}^{\chi \chi}(\vec{k}, \vec{k}+\vec{Q}, \omega)$ given in equations (A.2) and (A.3). Therefore, the $\alpha$ and $\beta$-band staggered magnetizations are expressed as:

$$
m_{f}^{\chi}=\sum_{\sigma} \sigma\left[\phi_{-\vec{Q} \sigma}^{\chi \chi} X_{3 \sigma}(\vec{Q})+\left|\kappa_{-\vec{Q}, \sigma}^{\beta \alpha}\right|^{2} \phi_{-\vec{Q} \sigma}^{\chi^{\prime} \chi^{\prime}} X_{2 \sigma}(\vec{Q})\right]
$$
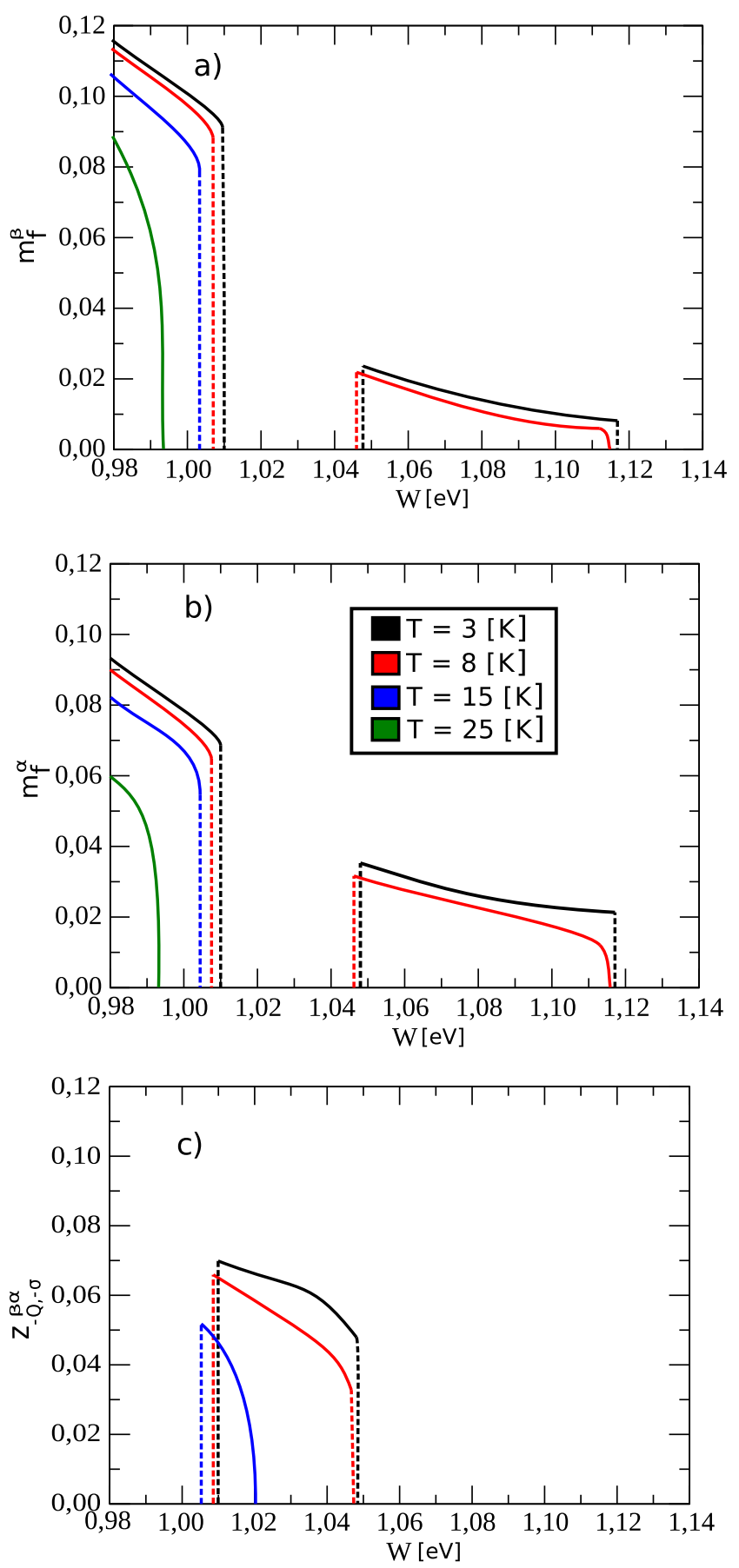

Figure 4. Behavior of the OPs as function of $W$ for different values of $\mathrm{T}$ at $h_{z}=0$.

where $\chi \neq \chi^{\prime}, \sigma=\uparrow, \downarrow$ corresponds to +- and

$$
\begin{aligned}
X_{3 \sigma}(\vec{Q})= & \frac{1}{N} \sum_{\vec{k}} \int_{C} \frac{\mathrm{d} \omega}{2 \pi i} f(\omega) \\
& \times \frac{\left(g_{\sigma}^{\chi^{\prime}}(\vec{k}, \omega) g_{\sigma}^{\chi^{\prime}}(\vec{k}+\vec{Q}, \omega)-\left(\phi_{-\vec{Q}_{\sigma}}^{\left.\chi^{\prime}\right)^{\prime}}\right)\right.}{D_{\vec{Q} \sigma}(\vec{k}, \omega)} .
\end{aligned}
$$

In equations (26), (27) and (30), $D_{\sigma}(\vec{k}, \vec{Q}, \omega)=0$ (see equation (A.4)) gives $E_{i, \sigma}(\vec{k})$ which are the quasi-particle 

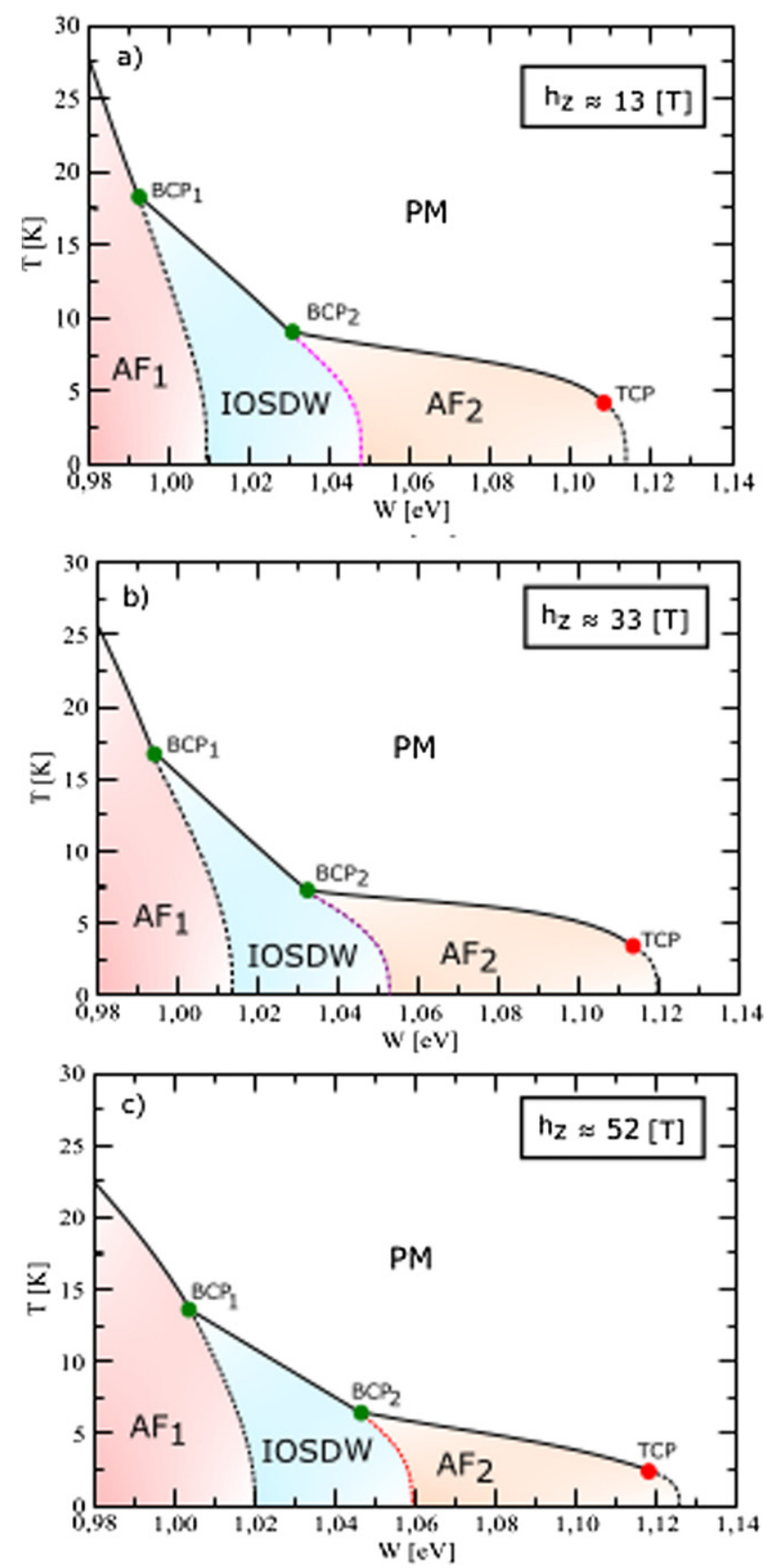

Figure 5. Phase diagram $T$ under $W$ for several values of $h_{z}$. The continuous line (black color) shows a second order transition while the discontinuous lines show a first-order transition.

dispersion relations that depend on the gaps $\phi_{-\vec{Q}, \sigma}^{\alpha \alpha}, \kappa_{-\vec{Q}, \sigma}^{\beta \alpha}$ and $\phi_{-\vec{Q}, \sigma}^{\beta \beta}$.

Within the Hartree-Fock approximation the total free energy is given by

$$
f_{t}=\Omega(T, \mu)+\mu N_{t}+f_{\mathrm{HF}}^{\mathrm{HO}}+f_{\mathrm{HF}}^{\mathrm{AF}}
$$

where $\mu$ is the chemical potential, $N_{t}=n_{\alpha}+n_{\beta}+n_{d}$ ( $n_{d}$ is the average occupation number of conduction electrons). Also

$$
\Omega(T, \mu)=-k_{\mathrm{B}} T \frac{1}{N} \sum_{\vec{k}} \sum_{j} \ln \left(1+\epsilon^{\frac{-\left(E_{j, \sigma}-\mu+H_{z}\right)}{k_{\mathrm{B}} T}}\right),
$$

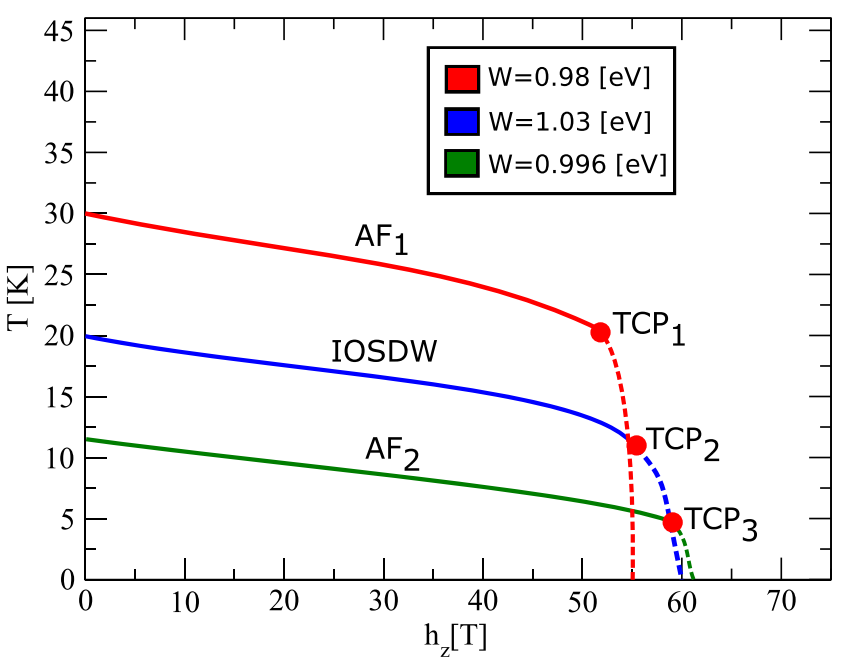

Figure 6. Phase diagram $T$ under $h_{z}$ for different $W$ values.

here $N$ is the total number of sites of the lattice. The remaining term is

$$
f_{\mathrm{HF}}^{\mathrm{HO}}=-N \sum_{\sigma} \sum_{\chi \neq \chi^{\prime}} z_{\vec{Q}, \sigma}^{\chi \chi^{\prime}} \kappa_{\vec{Q}, \sigma}^{\chi \chi^{\prime}}
$$

with $\sigma=\uparrow, \downarrow$. The term $f_{\mathrm{HF}}^{\mathrm{AF}}$ is given by

$$
f_{\mathrm{HF}}^{\mathrm{AF}}=N\left(U\left[\left(m_{f}^{\alpha}\right)^{2}+\left(m_{f}^{\beta}\right)^{2}\right]-2 J m_{f}^{\alpha} m_{f}^{\beta}\right)
$$

\section{Results}

The numerical calculations are performed with the nesting vector $\vec{Q}=(\pi / a, \pi / a, \pi / a)$ and assuming $V_{\alpha}(\vec{k})=V_{\alpha}$ and $\left\langle n_{f}^{\alpha}\right\rangle+$ $\left\langle n_{f}^{\beta}\right\rangle+\left\langle n_{d}\right\rangle=1.609$, where $\left\langle n_{d}\right\rangle$ is the average occupation of the conduction electrons. The occupation is chosen to enhance the PM phase instability and does not refer to any specific real $5 f$ system.

We have also chosen the following parameters: (i) the tightbinding parameters are $t_{d}=W_{d} / 6, t_{f}=W_{d} / 20, V_{\alpha}=1 / 10$ $\mathrm{eV}$ and $W_{f} / W_{d}=0.3$ where $2 W_{d(f)}$ is the width of the conduction $(5 f)$ band in order to be close to reference [9]. From now on, $W_{d}=W$. Our results are qualitatively robust to the numerical choice of parameters given above. The situation is more complicated when it comes to choosing the $J / U$ ratio. This point will be discussed below.

\subsection{Phases diagram without magnetic field}

The phases diagrams are constructed from the coupled equations for $m_{f}^{\alpha}, m_{f}^{\beta}, z_{-\vec{Q}, \sigma}^{\beta \alpha}$ and $z_{-\vec{Q},-\sigma}^{\beta \alpha}$ (see equations (25) and (29)) which must be solved self-consistently. In terms of OPs, $\mathrm{AF}_{1}$ and $\mathrm{AF}_{2}$ appears when $m_{f}^{\beta}>m_{f}^{\alpha}$ and $m_{f}^{\alpha}>m_{f}^{\beta}$, respectively. Both phases have $z_{-\vec{Q}, \sigma}^{\beta \alpha}=z_{-\vec{Q},-\sigma}^{\beta \alpha}=0$. The IOSDW phase has $z_{-\vec{Q}, \sigma}^{\beta \alpha}=-z_{-\vec{Q},-\sigma}^{\beta \alpha} \neq 0$ with $m_{f}^{\alpha}=m_{f}^{\beta}=0$. The locations of the first order transition lines are obtained using the free energy given in equations (31)-(34). 

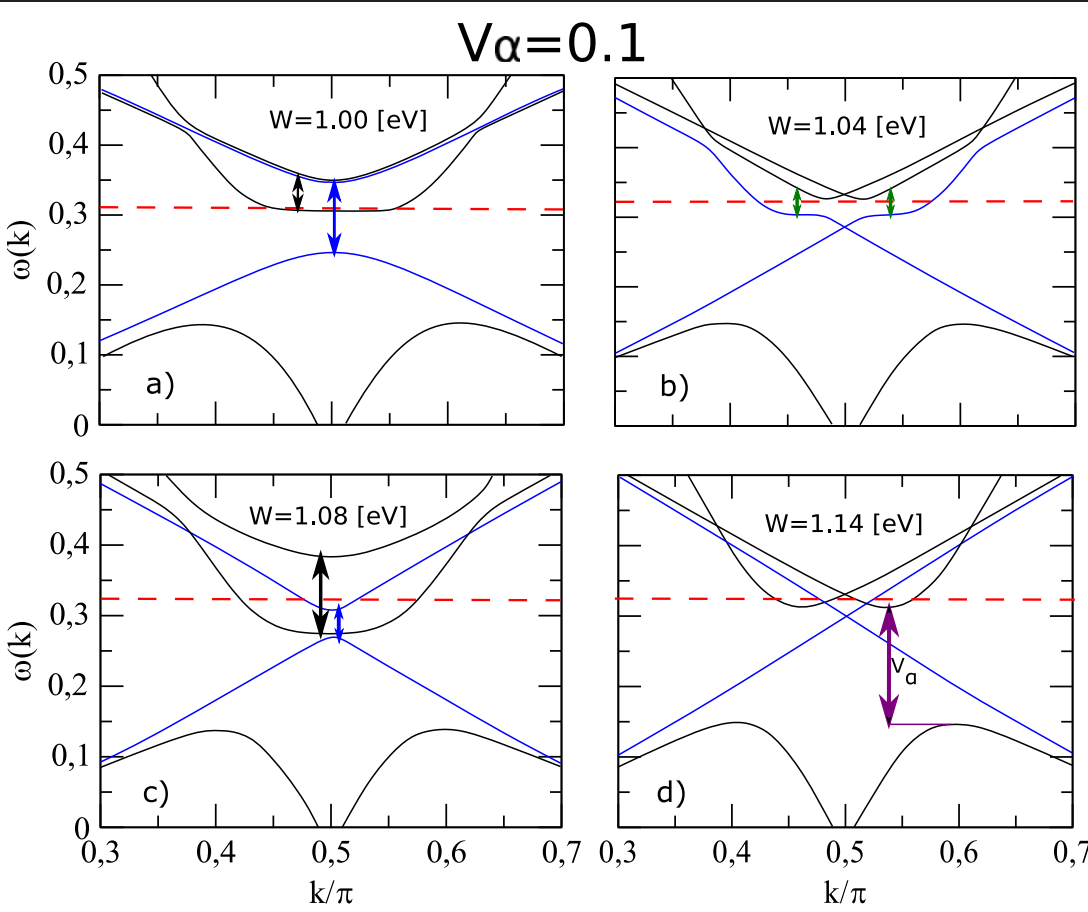

Figure 7. Quasiparticle dispersion relations for: (a) $W=1.00$, (b) $W=1.04$, (c) $W=1.08$, (d) $W=1.14$ in the absence of the magnetic field $h_{z}=0 \mathrm{~T}$ in the high symmetry direction $(0,0,0)-(1,1,1)$. The dashed red line indicates the position of the Fermi energy while the black and the blue lines show the $\alpha$ and $\beta$ bands, respectively.

In figure 1 , the phase diagram $J / U$ vs the bandwidth $W$ at $T=0$ is shown. For $J=0$, there is a complete decoupling between $z_{-\vec{Q}, \sigma}^{\beta \alpha}$ and $z_{-\vec{Q},-\sigma}^{\beta \alpha}$ as well as $m_{f}^{\alpha}$ and $m_{f}^{\beta}$ (see equations (25) and (29)). Although the $\mathrm{AF}_{1}$ and $\mathrm{AF}_{2}$ phases appear for certain $W$ ranges, the IOSDW phase does not exist for any $W$. When $J / U$ is finite but very small, the OPs recouple weakly. As a consequence, besides phases $\mathrm{AF}_{1}$ and $\mathrm{AF}_{2}$ the IOSDW phase begin to appear within a very small range of $W$. As $J / U$ increases, the width of the PM region within the phase diagram decreases. This behavior is accentuated until for a certain $J / U$ threshold, the PM phase disappears completely. This situation generates a PM dome, where above it there is a direct transition $\mathrm{AF}_{1} \rightarrow \mathrm{IOSDW} \rightarrow \mathrm{AF}_{2}$. In figure 2 is shown the behavior of the OPs illustrating the evolution of the phase diagram in figure 1. It should be noted that for $J=0$ (see figure 2(a)) the intermediate PM solution is more stable, although, $m_{f}^{\beta}>m_{f}^{\alpha}=0$. We remark that the presence of multicritical points in finite $T$ phase diagrams is entirely dependent on direct transitions between phases $\mathrm{AF}_{1}$, IOSDW and $\mathrm{AF}_{2}$ at $T=0$. Therefore, for finite $T$ diagrams, we will choose values of $J / U$ where the direct transition $\mathrm{AF}_{1} \rightarrow$ IOSDW $\rightarrow \mathrm{AF}_{2}$ appears at $T=0$.

Figure 3 displays the phase diagram $T$ vs $W$ for $J=U / 5$ with $U=0.165 \mathrm{eV}$. When the temperature is lowered, there are a second-order phase transitions from PM to any of phases $\mathrm{AF}_{1}, \mathrm{AF}_{2}$ or IOSDW. Moreover, when $W$ increases at lower $T$, the two magnetic phases $\mathrm{AF}_{1}$ and $\mathrm{AF}_{2}$, i.e., phases with time reversal symmetry breaking, are separated by the nonmagnetic IOSDW phases. It appears the phase transitions sequence $\mathrm{AF}_{1} \rightarrow \mathrm{IOSDW} \rightarrow \mathrm{AF}_{2}$, with first order line transitions separating the phases. To complete the sequence of phase transitions, there is a first order transition $\mathrm{AF}_{2} \rightarrow \mathrm{PM}$. Figure 4 shows the behavior of the POs as a function of the bandwidth $W$ for several finite temperature values. The behavior of the POs shown in this figure illustrates the phase evolution in the phase diagram shown in figure 3 . In the transition $\mathrm{AF}_{1} \rightarrow$ IOSDW both $m_{f}^{\beta}$ and $m_{f}^{\alpha}$ (with $m_{f}^{\beta}>m_{f}^{\alpha}$ ) collapse and the IOSDW becomes finite. In the transition IOSDW $\rightarrow \mathrm{AF}_{2}$ the opposite happens. The IOSDW OP collapses and $m_{f}^{\beta}$ and $m_{f}^{\alpha}$ are abruptly finite. But now with $m_{f}^{\beta}<m_{f}^{\alpha}$. Note that for $T=25 \mathrm{~K}$, there is only a second order transition $\mathrm{AF}_{1} \rightarrow \mathrm{PM}$.

\subsection{Phase diagrams with magnetic field}

In figure 5, the $T$ vs $W$ phase diagrams are shown with increasing values of $h_{z}$. The first significant effect is the lowering the critical temperatures corresponding to the three transitions $\mathrm{PM} \rightarrow \mathrm{AF}_{1}, \mathrm{PM} \rightarrow$ IOSDW and $\mathrm{PM} \rightarrow \mathrm{AF}_{2}$. This lowering of critical temperatures is more pronounced for the IOSDW and $\mathrm{AF}_{2}$ phases. Also, the locations of the first order lines in the phase transitions $\mathrm{AF}_{1} \rightarrow \mathrm{IOSDW} \rightarrow \mathrm{AF}_{2}$ and $\mathrm{AF}_{2} \rightarrow \mathrm{PM}$ are displaced to larger values of the $W$. These two effects compose what we will call from now on, flattening of phases. As consequence, there is a slight enlargement in the phase diagram of the $\mathrm{AF}_{1}$ region at the expense of IOSDW one. The IOSDW and $\mathrm{AF}_{2}$ regions also enlarge slight at the expense of $\mathrm{AF}_{2}$ and $\mathrm{PM}$ ones, respectively. We emphasize that there is a different size of these effects for each of the phases. Therefore, the $\mathrm{AF}_{2}$ phases are subjected to the most flattening.

In figure 6, we fix the values of $W$ in such way that we can evaluate the evolution of phases $\mathrm{AF}_{1}$, IOSDW and $\mathrm{AF}_{2}$ 
$\beta$
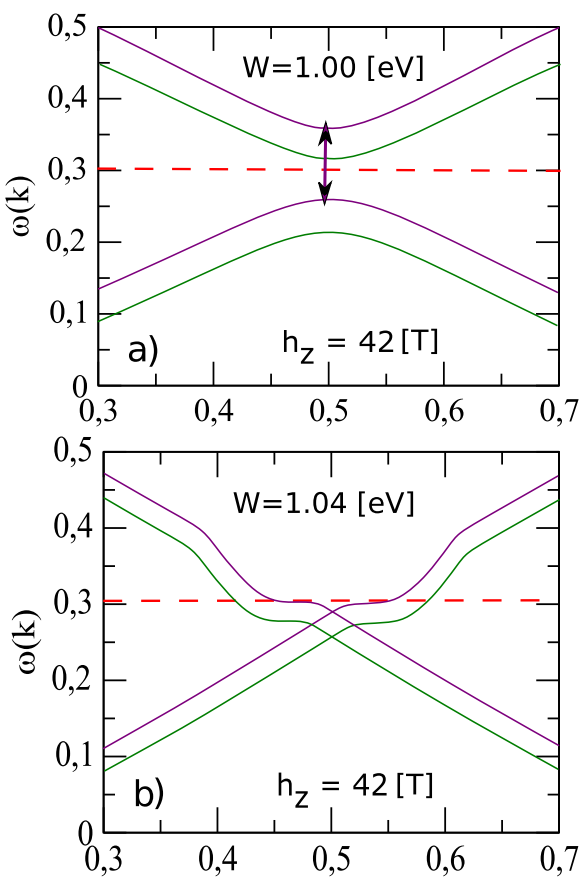

$0,70,3$
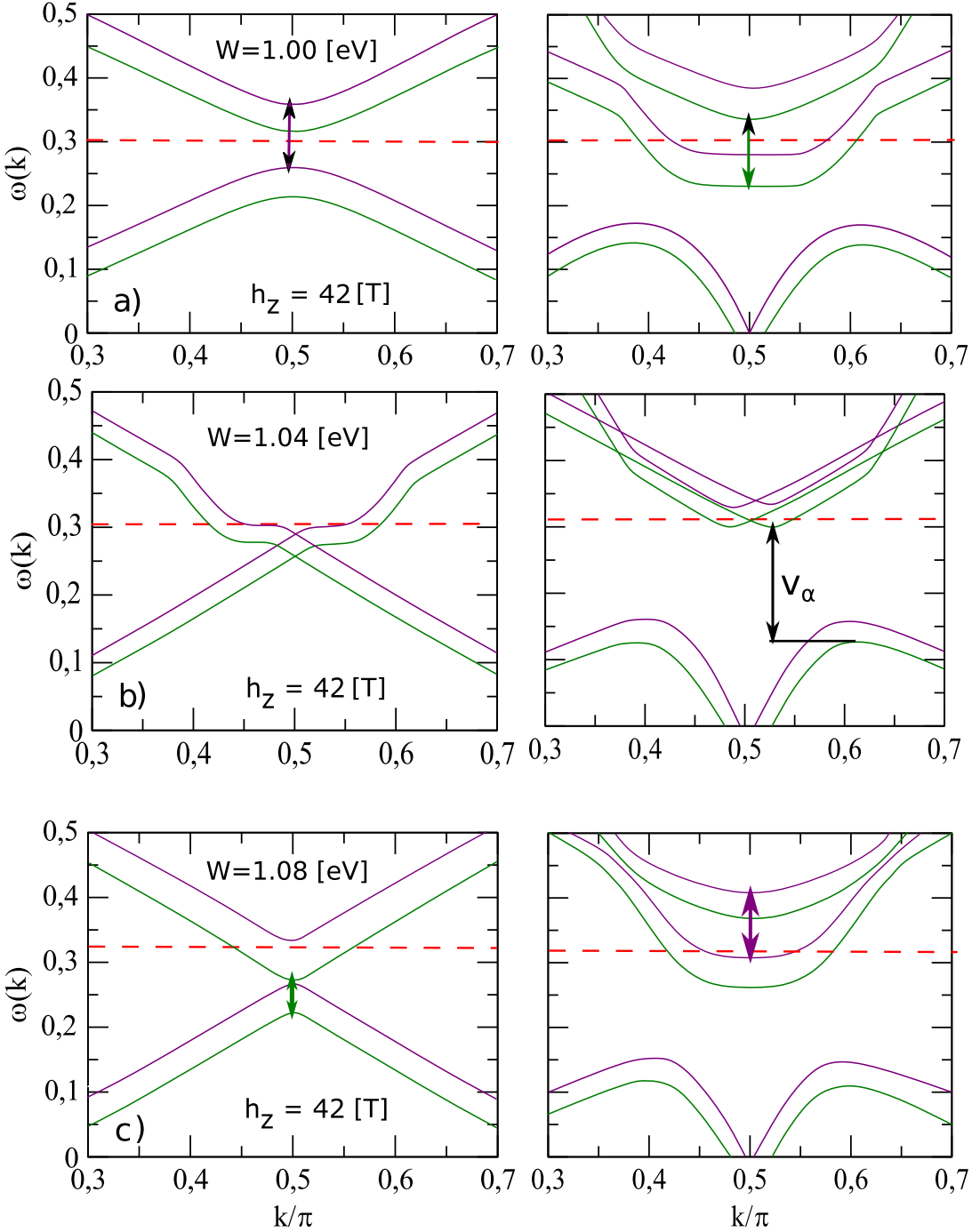

Figure 8. Quasiparticles dispersion relations at $T=0$ for two distinct values of $h_{z}$. The purple and green lines represent the spin up ( $\sigma=1$ ) and spin down $(\sigma=-1)$, respectively. Results are shown for the three phases $\mathrm{AF}_{1}(\mathrm{a}), \mathrm{IOSDW}_{(\mathrm{O})}$ and $\mathrm{AF}_{2}(\mathrm{c})$.

when $h_{z}$ is varied. We find that the three phases are completely suppressed above a certain value of $h_{z}$. However, there is a difference in the value of the suppression value of $h_{z}$ for each phase, i. e., the IOSDW and $\mathrm{AF}_{2}$ phases have almost the same suppression $h_{z}$ value while for $\mathrm{AF}_{1}$, the value is clearly smaller. Interestingly, our results indicate the same process of flattening observed in the $T$ vs $W$ surface occurs in the $T$ vs $h_{z}$ one. Again, the IOSDW and $\mathrm{AF}_{2}$ phases are more affected by process than $\mathrm{AF}_{1}$.

\subsection{Quasiparticles dispersion relations}

The quasiparticle dispersion relations $E_{i, \sigma}^{\chi}(\vec{k})$ for the bands $\alpha$ and $\beta$ are obtained from $D_{\sigma}(\vec{k}, \omega)=0$ (see appendix A). In absence of $h_{z}$, the evolution of $E_{i, \sigma}^{\chi}(\vec{k})$ for different $W$ at $T=0$ $\mathrm{K}$ is shown in figure 7. The case of the $\mathrm{AF}_{1}$ phase is shown in figure 7(a)). Here, the double arrows indicate approximately the locus of the $\beta$ and $\alpha$ gaps. Notice that the Fermi energy crosses both $\alpha$ and $\beta$ gaps. However, as an effect of the $V_{\alpha}$ hybridization, the $\alpha$ band crosses the Fermi energy near the gap, giving a semi-metallic character for the $\mathrm{AF}_{1}$ phase. In other words, the semi-metallicity refers to the situation where Fermi surface is reconstructed in only one of the bands. The same semi-metallic character is observed in the band structure of the IOSDW phase shown in figure 7(b). In contrast, the band structure for the $\mathrm{AF}_{2}$ (see figure 7(c)) phase indicates a metallic character.

Figure 8 displays $E_{i, \sigma}^{\chi}(\vec{k})$ with increasing $h_{z}$. Due to the spin dependence, $\sigma= \pm 1$, the number of bands is doubled. The semi-metallic nature of the $\mathrm{AF}_{1}$ phase, figure 8(a), and the metallic nature of the $\mathrm{AF}_{2}$ phase, figure $8(\mathrm{c})$, are maintained despite the increase in the $h_{z}$. On the other hand, in the IOSDW phase, figure 8(b), the increasing of the $h_{z}$ leads the system to a purely itinerant state (semi-metallic $\rightarrow$ metallic). In general, as the $h_{z}$ increases, the system enhances its itinerant electronic character, redistributing the Fermi surface, mainly due to the evolution from semi-metallic $\rightarrow$ metallic character of the IOSDW phase. 


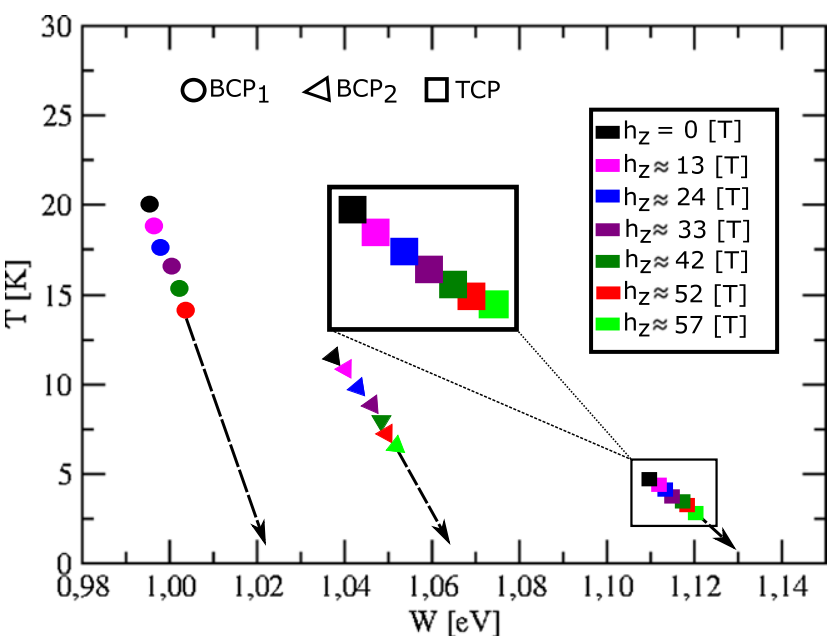

Figure 9. Evolution of the bicriticals points and TCP when $h_{z}$ increases.

\section{Multicritical points}

The sequence of first and second order phase transitions, that place the non-magnetic IOSDW phase between the $\mathrm{AF}_{1}$ and $\mathrm{AF}_{2}$ phases shown in figure 3 gives rise to two BCPs. The $\mathrm{BCP}_{1}$ is the meeting point of the second order transitions $\mathrm{PM} \rightarrow \mathrm{AF}_{1}$ and $\mathrm{PM} \rightarrow \mathrm{IOSDW}$ with the first order one $\mathrm{AF}_{1} \rightarrow$ IOSDW. While the second $\mathrm{BCP} \mathrm{BCP}_{2}$ involve $\mathrm{AF}_{2}$ instead of $\mathrm{AF}_{1}$. Moreover, there is also a TCP in the transition $\mathrm{AF}_{2} \rightarrow \mathrm{PM}$.

The locations of $\mathrm{BCP}_{1}, \mathrm{BCP}_{2}$ and TCP in figures 5(a)-(d)) when $h_{z}$ increases, reflects the process of flattening of the phases mentioned above. The effects of such process in the location of these multicritical points can be seen in the details in figure 9. The mentioned process appears in the shift of the positions of the multicritical points in $W$ as $h_{z}$ increases. It can be seen that the $\mathrm{BCP}_{1}$ is less shifted as compared to the $\mathrm{BCP}_{2}$ and the TCP. On the other hand, the displacement of the TCP is even more pronounced than that of $\mathrm{BCP}_{2}$.

In figure 6 , the phase transition lines feature three TCPs, $\mathrm{TCP}_{1}, \mathrm{TCP}_{2}$ and $\mathrm{TCP}_{3}$ which are related to the transition lines $\mathrm{AF}_{1} \rightarrow \mathrm{PM}$, IOSDW $\rightarrow \mathrm{PM}$ and $\mathrm{AF}_{2} \rightarrow \mathrm{PM}$, respectively. The process of flattening of the phases $\mathrm{AF}_{1}$, IOSDW and $\mathrm{AF}_{2}$ appears clearly in the ordering in $T$ and $h_{z}$ of each of the TCP's since $T_{\mathrm{TCP}_{1}}>T_{\mathrm{TCP}_{2}}>T_{\mathrm{TCP}_{3}}$ while $h_{z_{\mathrm{TCP}_{1}}}<h_{z \mathrm{TCP}_{2}}<h_{z \mathrm{TCP}_{3}}$.

\section{Conclusion}

This work has described, within a mean field approximation, the emergence of multicritical points coming from the competition among phases with OPs which have distinct parity properties (odd or even) under time reversal symmetry. We have used the UALM [13]. This model, suitable do describe $5 f$ electron systems, has two narrow bands, labeled by $\alpha$ and $\beta$, hybridized with a single wide conduction band. The interaction is given by the direct Columb and Hund's rule exchange terms. We obtain three distinct types of long-range order: (i) two conventional SDWs $\left(\mathrm{AF}_{1}\right.$ and $\left.\mathrm{AF}_{2}\right)$ and, (ii) the non-magnetic
IOSDW. This exotic phase is described by a purely imaginary OP that mixes the $\alpha$ and $\beta$ bands. The conventional SDWs are described by the real staggered magnetization of each band $m_{f}^{\alpha}$ and $m_{f}^{\beta}$, where $\mathrm{AF}_{1}$ and $\mathrm{AF}_{2}$ are defined by $m_{f}^{\beta}>m_{f}^{\alpha}$ and $m_{f}^{\alpha}>m_{f}^{\beta}$, respectively. It is worth mentioning that the existence of a non-magnetic SDW has been suggested in other context such as iron superconductors [21].

The competition among phases takes place with the variation of the $W$ (mimicking the pressure variation) and $h_{z}$. In the absence of $h_{z}$, the phase diagram $T$ vs $W$ displays at low $T$ a sequence of first order phase transitions $\mathrm{AF}_{1} \rightarrow \mathrm{IOSDW} \rightarrow \mathrm{AF}_{2}$. We also found two $\mathrm{BCP}$. The first one, called $\mathrm{BCP}_{1}$, is the intersection of the second order line transitions $\mathrm{PM} \rightarrow \mathrm{AF}_{1}$ and $\mathrm{PM} \rightarrow \mathrm{IOSDW}$ with the first order one $\mathrm{AF}_{1} \rightarrow$ IOSDW. For the second $\mathrm{BCP}$, called $\mathrm{BCP}_{2}, \mathrm{AF}_{1}$ is replaced by $\mathrm{AF}_{2}$. Lastly, there is a TCP in the transition $\mathrm{AF}_{2} \rightarrow \mathrm{PM}$. The location of the BCPs indicates that their existence is a direct result of the distinct parity property under time reversal symmetry of the phases $\mathrm{AF}_{1}, \mathrm{AF}_{2}$ and IOSDW. This is in agreement with general arguments based on a Landau free energy expansion.

When $h_{z}$ is turned on, there are important changes in the IOSDW phase and more markedly in the $\mathrm{AF}_{2}$ one. These two phases flatten out which means that they stabilize at lower $T$ but with higher $W$ values as compared to the situation without $h_{z}$. This is reflected in the location of $\mathrm{BCP}_{2}$ and TCP. The evolution of their locations with the field shows the tendency for these multicritical points to disappear because of the flattening process of $\mathrm{AF}_{2}$ and IOSDW. The different behavior of the phases when applying $h_{z}$ is related to the very nature of each one. Phase $\mathrm{AF}_{1}$ is semimetallic since the Fermi surface constructs in only one of the bands. In contrast, the phase $\mathrm{AF}_{2}$ is totally metallic. These two phases retain the same nature when the $h_{z}$ is applied. Nevertheless, the IOSDW phase change its nature when the field is applied. While one of the bands always has a Fermi surface, the other band has a totally constructed Fermi surface. The gradual change from semimetallic to metallic is the ultimate cause that leads the IOSDW phase to have the flattening process more accentuated than $\mathrm{AF}_{1}$ phase, although not as much as the $\mathrm{AF}_{2}$ one.

To conclude, although our results refer to a specific model, we believe that the evolution of multicritical points with $W$ and $h_{z}$ as described here may be more general. For instance, motivated by the concept of adiabatic continuity [22], one may suggest the possibility that the present problem with three POs (two of them reals and one purely imaginary) can be described in a unified way in a single PO. That would be similar to the interesting proposal made by Haule and Kotliar that a complex PO accounts for the behavior of $\mathrm{URu}_{2} \mathrm{Si}_{2}$ under $W$ and $h_{z}$ [23]. In such scenario, it would be necessary to re-interpret the multicritical points. We are currently investigating this possibility.

\section{Acknowledgments}

The present study was supported by the Brazilian agencies Conselho Nacional de Desenvolvimento Científico e 
Tecnológico (CNPq) and CAPES. SGM also acknowledge the Internationalization Program (PRINT) of Universidade Federal do Rio Grande do Sul and the hospitality of Temple University.

\section{Data availability statement}

All data that support the findings of this study are included within the article (and any supplementary files).

\section{Appendix A. Green functions}

The Green functions necessary to obtain the IOSDW and AF OPs can be obtained directly from the equation (16). Therefore, $G_{f, \sigma}^{\beta \alpha}(\vec{k}, \vec{k}+\vec{Q}, \omega)$ is given as

$$
\begin{aligned}
G_{f, \sigma}^{\beta \alpha}(\vec{k}, \vec{k}+\vec{Q}, \omega)= & D_{\sigma}^{-1}(\vec{k}, \vec{Q}, \omega) \times\left[\left|\kappa_{-\vec{Q}, \sigma}^{\beta \alpha}\right|^{3}\right. \\
& \left.+\left|\phi_{-\vec{Q}, \sigma}^{\alpha \alpha}\left\|\kappa_{-\vec{Q}, \sigma}^{\beta \alpha}\right\| \phi_{-\vec{Q}, \sigma}^{\beta \beta}\right|\right] \\
& -\left(\omega-E_{f, \sigma}^{\beta}(\vec{k}+\vec{Q})\right)\left|\kappa_{-\vec{Q}, \sigma}^{\beta \alpha}\right| \\
& \times\left(\omega-E_{f, \sigma}^{\alpha}(\vec{k}+\vec{Q})-\xi^{\alpha}(\vec{k}+\vec{Q})\right) .
\end{aligned}
$$

While $G_{f, \sigma}^{\alpha \alpha}(\vec{k}, \vec{k}+\vec{Q}, \omega)$ and $G_{f, \sigma}^{\beta \beta}(\vec{k}, \vec{k}+\vec{Q}, \omega)$ are:

$$
\begin{aligned}
G_{f, \sigma}^{\alpha \alpha}(\vec{k}, \vec{k}+\vec{Q} \omega)= & D_{\sigma}^{-1}(\vec{k}, \vec{Q}, \omega) \times\left[\left|\phi_{-\vec{Q}, \sigma}^{\beta \beta}\right|^{2}\left|\phi_{-\vec{Q}, \sigma}^{\alpha \alpha}\right|\right. \\
& -\left(\omega-E_{f, \sigma}^{\beta}(\vec{k}+\vec{Q})\right)\left|\phi_{-\vec{Q}, \sigma}^{\alpha \alpha}\right| \\
& \left.\times\left(\omega-E_{f, \sigma}^{\beta}(\vec{k})\right)-\left|\kappa_{-\vec{Q}, \sigma}^{\beta \alpha}\right|^{2}\left|\phi_{-\vec{Q}, \sigma}^{\beta \beta}\right|\right]
\end{aligned}
$$

and

$$
\begin{aligned}
& G_{f, \sigma}^{\beta \beta}(\vec{k}, \vec{k}+\vec{Q}, \omega)=D_{\sigma}^{-1}(\vec{k}, \vec{Q}, \omega) \\
& \quad \times\left[\left|\phi_{-\vec{Q}, \sigma}^{\alpha \alpha}\right|^{2}\left|\phi_{-\vec{Q}, \sigma}^{\beta \beta}\right|-\left(\omega-E_{f, \sigma}^{\alpha}(\vec{k})-\xi^{\alpha}(\vec{k})\right)\left|\phi_{-\vec{Q}, \sigma}^{\beta \beta}\right|\right. \\
& \left.\quad-\left(\omega-E_{f, \sigma}^{\alpha}(\vec{k}+\vec{Q})-\xi^{\alpha}(\vec{k}+\vec{Q})\right)\left|\kappa_{-\vec{Q}, \sigma}^{\beta \alpha}\right|^{2}\left|\phi_{-\vec{Q}, \sigma}^{\alpha \alpha}\right|\right] .
\end{aligned}
$$

The term $D_{\sigma}(\vec{k}, \vec{Q}, \omega)$ in equations (A.1)-(A.3) is explicitly given as:

$$
\begin{aligned}
D_{\sigma}(\vec{k}, \vec{Q}, \omega)= & {\left[\left(\omega-E_{f \sigma}^{\alpha}(\vec{k})-\xi^{\alpha}(\vec{k})\right)\left(\omega-E_{f \sigma}^{\beta}(\vec{k})\right)\right.} \\
& \times\left(\omega-E_{f \sigma}^{\alpha}(\vec{k}+\vec{Q})-\xi^{\alpha}(\vec{k}+\vec{Q})\right) \\
& \times\left(\omega-E_{f \sigma}^{\beta}(\vec{k}+\vec{Q})\right]-\left|\kappa_{-\vec{Q}, \sigma}^{\alpha \beta}\right|^{2}\left(\omega-E_{f \sigma}^{\alpha}(\vec{k})\right. \\
& \left.-\xi^{\alpha}(\vec{k})\right)\left(\omega-E_{f \sigma}^{\beta}(\vec{k}+\vec{Q})\right)-\left|\kappa_{\vec{Q}, \sigma}^{\beta \alpha}\right|^{2} \\
& \times\left(\omega-E_{f \sigma}^{\beta}(\vec{k})\right)\left(\omega-E_{f \sigma}^{\alpha}(\vec{k}+\vec{Q})-\xi^{\alpha}(\vec{k}+\vec{Q})\right)
\end{aligned}
$$

$$
\begin{aligned}
& -\left(\phi_{-\vec{Q}, \sigma}^{\beta \beta}\right)^{2}\left(\omega-E_{f \sigma}^{\alpha}(\vec{k})-\xi^{\alpha}(\vec{k})\right)\left(\omega-E_{f \sigma}^{\alpha}\right. \\
& \left.(\vec{k}+\vec{Q})-\xi^{\alpha}(\vec{k}+\vec{Q})\right)-\left(\phi_{-\vec{Q}, \sigma}^{\alpha \alpha}\right)^{2}\left(\omega-E_{f \sigma}^{\beta}(\vec{k})\right) \\
& \times\left(\omega-E_{f \sigma}^{\beta}(\vec{k}+\vec{Q})\right)+\left(\phi_{-\vec{Q}, \sigma}^{\alpha \alpha}\right)^{2}\left(\phi_{-\vec{Q}, \sigma}^{\beta \beta}\right)^{2} \\
& -\left(\phi_{-\vec{Q}, \sigma}^{\alpha \alpha}\right)\left(\phi_{-\vec{Q}, \sigma}^{\beta \beta}\right)\left(\left|\kappa_{\vec{Q}, \sigma}^{\beta \alpha}\right|^{2}+\left|\kappa_{-\vec{Q}, \sigma}^{\alpha \beta}\right|^{2}\right) \\
& +\left|\kappa_{\vec{Q}, \sigma}^{\beta \alpha}\right|^{2}\left|\kappa_{-\vec{Q}, \sigma}^{\alpha \beta}\right|^{2} .
\end{aligned}
$$

\section{ORCID iDs}

Julián Faúndez (D) https://orcid.org/0000-0002-6909-0417 S G Magalhães (i) https://orcid.org/0000-0002-6874-7579

E J Calegari (D) https://orcid.org/0000-0002-0157-7835

P S Riseborough (D) https://orcid.org/0000-0002-2216-3586

\section{References}

[1] Stillwell R L, Liu I-L, Harrison N, Jaime M, Jeffries J R and Butch N P 2017 Phys. Rev. B 95014414

[2] Shrestha K, Antonio D, Jaime M, Harrison N, Mast D S, Safarik D, Durakiewcz T, Griveau J-C and Gofric K 2017 Sci. Rep. 76642

[3] Vališka M et al 2018 Phys. Rev. B 98174439

[4] Correa V F et al 2012 Phys. Rev. Lett. 109246405

[5] Niklowitz P G, Pfleiderer C, Keller T, Vojta M, Huang Y-K and Mydosh J A 2010 Phys. Rev. Lett. 106106406

[6] Belitz D and Kirkpatrick T R 2017 Phys. Rev. Lett. 119 267202

[7] Gegenwart P, Si Q and Steglich F 2008 Nat. Phys. 4186

[8] Misawa T, Yamaji Y and Imada M 2008 J. Phys. Soc. Japan 77 093712

[9] Magalhaes S G, Lausmann A C, Calegari E J and Riseborough P S 2020 Phys. Rev. B 101064407

[10] Caroli B, Caroli C and Fredkin D R 1969 Phys. Rev. 178 599

[11] Perkins N B, Nunez-Regueiro M D, Iglesias J R and Coqblin B 2007 Phys. Rev. B 76125101

[12] Thomas C, da Rosa Simões A S, Iglesias J R, Lacroix C, Perkins N B and Coqblin B 2011 Phys. Rev. B 83014415

[13] Riseborough P S, Coqblin B and Magalhaes S G 2012 Phys. Rev. B 85165116

[14] Schoenes J 1980 Phys. Rep. 66187

[15] Havela L, Sechovsky V, de Boer F R, Brück E and Nakotte H 1992 Physica B 177159

[16] Maskova S et al 2019 Phys. Rev. B 99064415

[17] Mineev V P and Zhitomirski M E 2005 Phys. Rev. B 72 014432

[18] Shah N, Chandra P, Coleman P and Mydosh J A 2000 Phys. Rev. B 61564

[19] Chaikin P M and Lubensky T C 1995 Principles of Condensed Matter Physics (Cambridge: Cambridge University Press)

[20] Calegari E J, Magalhaes S G and Riseborough P S 2017 npj Quantum Mater. 248

[21] Rodriguez J P 2020 Phys. Rev. B 102024521

[22] Jo Y J, Balicas L, Capan C, Behnia K, Lejay P, Flouquet J, Mydosh J A and Schlottmann P 2007 Phys. Rev. Lett. 98 166404

[23] Haule K and Kotliar G 2010 Europhys. Lett. 8957006 